\title{
Advantages of Interval Laparoscopic Appendectomy for Periappendiceal Abscess
}

\author{
Gilbert Young Jin Kim, M.D., Sun Hyong You, M.D., Bong-Hyeon Kye, M.D., Taek Su Kwon, M.D., \\ Yoon Suk Lee, M.D., Seong Taek Oh, M.D., Jun-Gi Kim, M.D., In Kyu Lee, M.D. \\ Department of Surgery, The Catholic University of Korea College of Medicine, Seoul, Korea
}

Purpose: Laparoscopic appendectomy is a common procedure for treatment of appendicitis. However, in some complicated cases, like periappendiceal abscess, deciding on treatment options is very challenging. Early appendectomy or interval appendectomy may be possible, but remains controversial.

Methods: We prospectively studied the advantages of interval appendectomy in 21 patients with periappendiceal abscess using a laparoscopic method versus 14 patients with immediate initial laparotomy.

Results: In the interval appendectomy group (INT group), in periappendiceal abscess, use of a laparoscopic method was advantageous in terms of operation time $(p<0.001)$, less fasting time $(p<0.001)$, and fewer postoperative complications $(p=0.032)$. However, the total cost in the INT group was

\section{INTRODUCTION}

In 1886, Reginald Fitz ${ }^{1}$ established the terminology of appendicitis, which is the primary cause of right lower quadrant inflammation. In 1889, Chester McBurney described the surgical treatment of acute appendicitis that early surgical laparotomy has been the standard of treatment for appendicitis. Approximately $7 \%$ of the population in the Western countries experience appendicitis and annually, 10 in 10,000 receive an appendectomy during their lifetime., ${ }^{2,3}$ Although there has been an improvement in imaging modalities, such as ultrasonography and CT scanning, the rate of misdiagnosis has not yet declined $(15.5 \%) .^{4}$ Misdiagnosis or delayed clinical impressions can cause the inflammation to progress into perforated appendicitis or periappendiceal abscess which results in clinical crisis. Worldwide, perforated appendicitis is the leading cause of death in general surgery.

Received June 2, 2014, Revised July 18, 2014,

Accepted July 28, 2014

※ Corresponding author: In Kyu Lee

Department of Surgery, Yeouido St. Mary's Hospital, The Catholic University of Korea College of Medicine, 62, Yeouido-dong, Yeongdeungpo-gu, Seoul 150-713, Korea.

Tel : +82-2-3779-1099, Fax : +82-2-786-0802

E-mail : cmcgslee@catholic.ac.kr
$1,686,000 \pm 940,000$ South Korean won (KRW) compared with $1,506,000 \pm 322,000 \mathrm{KRW}$ in the early appendectomy group (EAR group) $(p=0.007)$ because patients in the INT group required two hospital visits. The total length of hospital stay postoperatively, was $7.31 \pm 2.726$ days in the INT group, compared with $9.21 \pm 3.378$ days in the EAR group $(p=0.537)$.

Conclusion: We recommend interval appendectomy as the preferable approach for the periappendiceal abscess, as it can result in more favorable postoperative surgical outcomes, fewer complications, and less antibiotic usage.

Key words: Interval appendectomy, Laparoscopic appendectomy, Periappendiceal abscess, Percutaneous drainage, Cost effectiveness
Laparoscopic appendectomy was first reported by gynecologist Kurt Semm in $1983 .{ }^{5}$ Since then, the number performed procedures to treat acute appendicitis have continuously increased. However, complicated cases of appendicitis, such as perforated appendicitis or periappendiceal abscess, remain surgical concern, and the treatment become controversial.

\section{MATERIALS AND METHODS}

This study was undertaken prospectively. Patients who visited Yeouido St. Mary's Hospital, South Korea, with right lower quadrant abdominal pain in between May 2009 to August 2011 were considered for this study. Total of 35 patients were finally enrolled from a group of 39 candidates based on the diagnosis of periappendiceal abscess, which was made based on abdominal CT scans. Three participants, who were younger than 12 years old and one participant with severe co-morbidity (ASA score IV) were excluded. Two different surgical teams participated in this study: one group preformed interval appendectomy (INT group), and the other group preformed early appendectomy (EAR group). 21 patients in the INT group, 16 patients actually received interval appendectomy in which, categorized as INT-A group. Within this group, 14 patients received early appendectomy. 


\section{1) Treatment}

In certain cases in the INT group, abscess drainage was deemed appropriate without damaging the adjacent small bowels and the major vessels that when, abscess cavity was more than $3 \mathrm{~cm}$ in size, interventional abscess aspiration or abscess drainage (pig-tail catheter) was performed. The patients were discharged when they could tolerate a soft diet and the abdominal pain was relieved without any fever. Intravenous antibiotics were administered during the hospital stay using third-generation cephalosporins combined with metronidazole. IV antibiotics were changed to oral antibiotics in consideration for diet and the discharge from the hospital while maintaining antibiotics until abdominal pain was reduced. In the INT group, follow-up abdominal CT was performed one month after the initial visit. All of the patients who were enrolled in the INT group, clinicians recommended interval appendectomy on an outpatient basis. Some patients who refused the surgery, the clinician decided to observe without operation at an outpatient clinic. In the INT group, laparoscopic surgery was our goal for interval appendectomy; in the EAR group, the surgeon decided whether to use a laparoscopic method or open method.

\section{2) Definitions and statistical analysis}

The Periappendiceal abscess was defined as fluid collection adjacent to the appendix with attenuation of $0 \sim 20 \mathrm{HU}$ on CT scans. We measured the size of the abscess as the longest length of the abscess cavity. Follow-up abdominal CT scanning was performed, one month after the initial CT scan to assess the response to initial nonoperative treatment in the INT group. Response was defined as follows: complete response denoted full disappearance of the abscess cavity and inability to detect the initial abscess cavity on the abdominal CT scan; partial response denoted partial regression of the initial abscess cavity; and non-response denoted as no change in the abscess cavity, progression of the abscess size, or rupture of the abscess cavity on CT scan images.

Clinical characteristics were compared between the two groups. The results of the continuous variables are expressed as the mean \pm standard deviation. Univariate statistical analysis was performed using the Mann-Whitney $U$ test for continuous independent samples and the $\mathrm{X}^{2}$ test or Fisher exact test for categorical variables. The statistical analyses were performed using SPSS for Windows, version 12.0 (SPSS Inc., Chicago, IL, USA), and $p<0.05$ was considered to indicate a statistically significant difference with a $95 \%$ confidence interval.

This study was approved by the institutional review board of the ethical committee of the College of Medicine of the Catholic University of Korea (SC12RISI0069).

\section{RESULTS}

\section{1) Patient characteristics}

A flow diagram for patient enrolled in each group is provided in Fig. 1. Twenty-one patients were enrolled in the INT group, who underwent interval laparoscopic appendectomy. INT group received nonoperative management, by staying NPO (Nothing Per Os) with IV antibiotics and hydration. In this group, nine patients $(42.9 \%)$ received pig-tail catheter drainage or aspiration in the Department of Radiology. The radiological findings were appropriate for interventional pig-tail drainage without damaging the adjacent bowel or blood vessels. In our study, 9 out of $21(42.9 \%)$ patients showed partial response, and 10 out of $21(47.6 \%)$ showed complete response with disappearance of the initial abscess cavity. Only two patients showed nonresponse during the follow-up period. Among these 21 patients, 16 patients received interval appendectomy, three months after their initial treatment. Among the patients who showed partial or complete response, four refused to receive interval appendectomy since, there were no symptoms of abdominal pain or tenderness, without fever. However, we recommended planned appendectomy and warned the patients about the potential recurrence of inflammation.

Age, gender, leukocyte count, ESR (Erythrocyte Sedimentation Rate), CRP (C-Reactive Protein), abscess size, and onset of first symptom at initial visit, showed little differences between the two groups (Table 1). There were no differences in the recovery time from fever $\left(<37^{\circ} \mathrm{C}\right)$ between the groups; specifically, the duration was $2.90 \pm 3.687$ days in the INT group and $2.29 \pm 2.463$ days in the EAR group $(p=0.168)$. The length of fasting (in days) was longer in the EAR group (3.50 \pm 3.276 ) compared with the INT group $(3.33 \pm 1.528 ; p=0.168)$, indicating that the INT group started the diet earlier than the EAR group (Table 2).

\section{2) Comparison of clinical features associated with surgery between the 2 groups}

Fifteen out of 16 patients underwent laparoscopic approach in the INT-A group (93.75\%). One patient who underwent a conventional open approach appendectomy was diagnosed with carcinomatosis peritonei of unknown origin based on the follow-up abdominal CT scan. 12 out of the 14 patients in the EAR group (85.7\%) underwent laparoscopic approach (Table 3).

Types of operation differed according to clinical significance. 


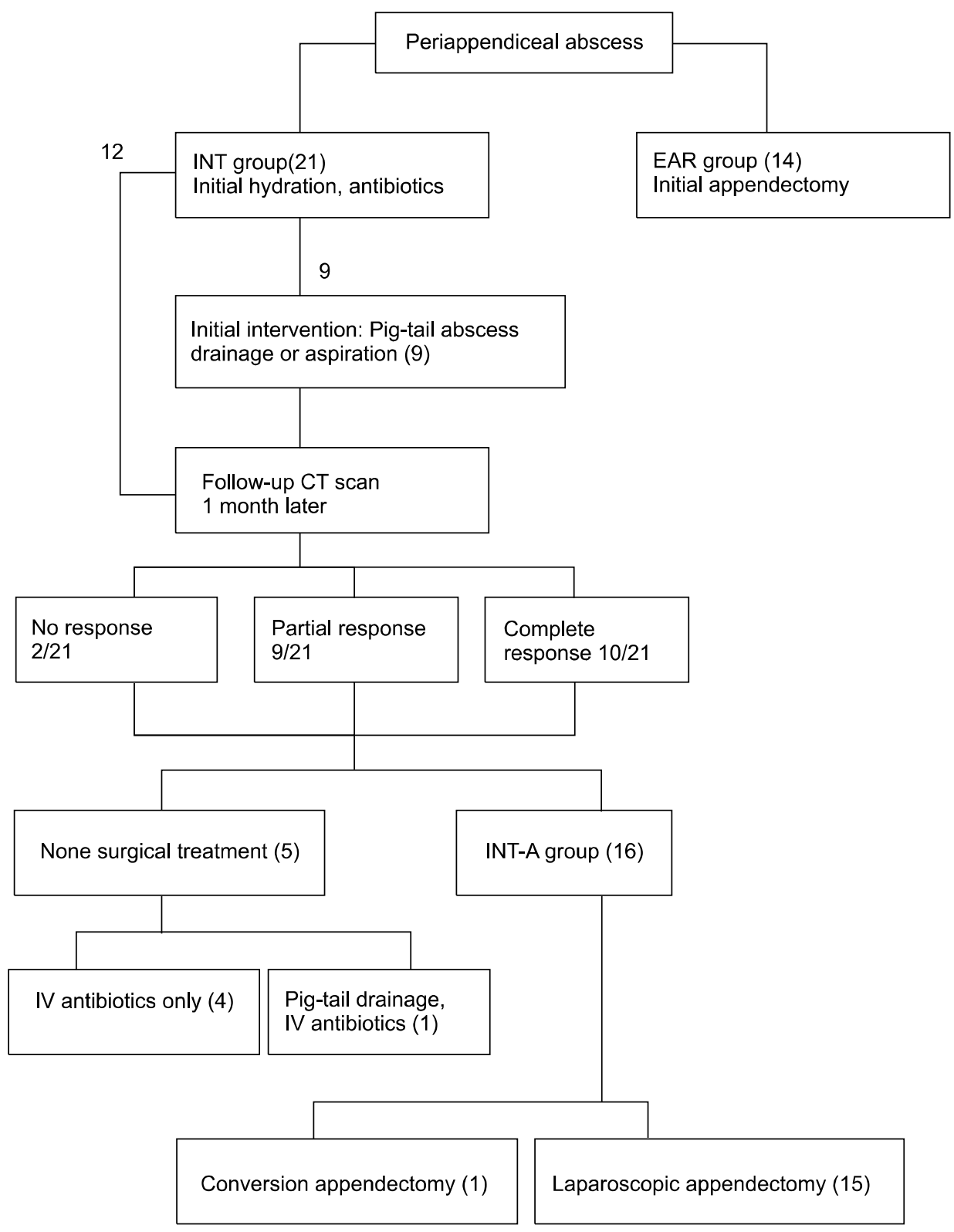

Fig. 1. Flow diagram of the treatment schedule in the INT and EAR groups.

Appendectomies were performed in 14 of the 16 patients $(87.5 \%)$ in the INT-A group and in 12 of the 14 patients $(85.7 \%)$ in the EAR group. Extended operations were performed in two patients in each group. In the INT-A group, two patients underwent cecal wedge resection, and in the EAR group, one patient underwent cecal wedge resection, and another patient underwent appendectomy with adnexectomy. The operation time was shorter in the INT group $(75.44 \pm 23.49 \mathrm{~min})$ compared with the EAR group $(112.14 \pm 62.9 \mathrm{~min} ; p<0.001)$ (Table 4).

Placement of a surgical drain (JP drain) after surgery was more frequent in the EAR group, with 13 cases (92.85\%), than in the INT-A group, with 9 cases $(56.25 \%$; $p=0.039)$, which indicates the need of surgical drain placement as follow; difficult to remove abscess cavity, incomplete removal of the infected material in the abscess cavity and intraoperative bleeding. However, the mean duration of surgical drain placement was not significantly different between the two groups (EAR group, 3.78 days; INT-A group, 5.23 days; $p=0.520$ ).

The number of fasting days after surgery was significantly shorter in the INT-A group $(1.90 \pm 1.091)$ compared with the EAR group (3.5 \pm 3.276 days; $p<0.001)$, indicating that a faster recovery of a normal diet is achieved with interval appendectomy.

The length of the initial hospital stay in patients admitted for interval appendectomy in the INT group was $7.31 \pm 2.726$ days, 
Table 1. Comparison of patient characteristics between the INT and EAR groups

\begin{tabular}{lccc}
\hline & $\begin{array}{c}\text { INT group } \\
(\mathrm{n}=21)\end{array}$ & $\begin{array}{c}\text { EAR group } \\
(\mathrm{n}=14)\end{array}$ & $p$ value \\
& $53.48 \pm 19.5$ & $57.71 \pm 17.7$ & 0.518 \\
Age (years) & & & \\
Gender & $11(52.4 \%)$ & $6(42.9 \%)$ & \\
Female & $10(47.6 \%)$ & $8(57.1 \%)$ & \\
Male & $11,100 \pm 3,250$ & $10,900 \pm 3,280$ & 0.865 \\
Initial leukocyte count & & & \\
$\quad\left(/ \mathrm{mm}^{3}\right)$ & $64.15 \pm 35.5$ & $53.31 \pm 26.9$ & 0.356 \\
ESR (mm/h) & $100 \pm 80.4$ & $132 \pm 85.4$ & 0.280 \\
CRP (mg/dl) & $44.5 \pm 24.1$ & $30.6 \pm 17.1$ & 0.083 \\
Abscess size (mm) & $20.57 \pm 38.5$ & $5.57 \pm 3.9$ & 0.158 \\
Symptom onset (days) & & \\
\hline
\end{tabular}

ESR $=$ Erythrocyte Sedimentation Rate, CRP $=$ C-reative protein.

Table 2. Comparison of initial treatment outcomes between the INT-A and EAR groups

\begin{tabular}{lccc}
\hline & $\begin{array}{c}\text { INT-A group } \\
(\mathrm{n}=16)\end{array}$ & $\begin{array}{c}\text { EAR group } \\
(\mathrm{n}=14)\end{array}$ & $p$ value \\
\hline $\begin{array}{l}\text { Recovery time for normal } \\
\text { temperature (days) }\end{array}$ & $2.90 \pm 3.687$ & $2.29 \pm 2.463$ & 0.168 \\
$\begin{array}{l}\text { Duration of IV antibiotics } \\
\text { (days) }\end{array}$ & $6.81 \pm 2.909$ & $7.36 \pm 2.706$ & 0.939 \\
$\begin{array}{l}\text { Duration of fasting (days) } \\
\text { Duration of hospitalization } \\
\text { (days) }\end{array}$ & $3.33 \pm 1.528$ & $3.50 \pm 3.276$ & 0.004 \\
\hline
\end{tabular}

In the INT group, the patients received initial nonoperative treatment; in the EAR group, the patients received initial appendectomy or extended operation.

Table 3. Comparison of operation types between the INT-A and EAR groups

\begin{tabular}{lccc}
\hline & $\begin{array}{c}\text { INT-A group } \\
(\mathrm{n}=16)\end{array}$ & $\begin{array}{c}\text { EAR group } \\
(\mathrm{n}=14)\end{array}$ & $p$ value \\
\hline $\begin{array}{l}\text { Operation approach methods } \\
\text { Laparoscopy }\end{array}$ & & & \\
$\quad 15(93.75 \%)$ & $12(85.7 \%)$ & 0.157 \\
Open & $1(6.25 \%)$ & $2(14.3 \%)$ & \\
Operation methods & & & \\
Appendectomy & $14(87.5 \%)$ & $12(85.7 \%)$ & 0.743 \\
Extended operation & $2(12.5 \%)$ & $2(14.3 \%)$ & \\
$\quad$ Cecal wedge resection & $2(12.5 \%)$ & $1(7.1 \%)$ & \\
$\quad \begin{array}{l}\text { Appendectomy+ } \\
\quad \text { adnexectomy }\end{array}$ & & $1(7.1 \%)$ & \\
\hline
\end{tabular}

Table 4. Comparison of postoperative outcomes between the INT-A and EAR groups

\begin{tabular}{lccc}
\hline & $\begin{array}{c}\text { INT-A group } \\
(\mathrm{n}=16)\end{array}$ & $\begin{array}{c}\text { EAR group } \\
(\mathrm{n}=14)\end{array}$ & $p$ value \\
\hline $\begin{array}{l}\text { Operation time (min) } \\
\text { JP drain insertion }\end{array}$ & $75.44 \pm 23.49$ & $112.14 \pm 62.90$ & 0.000 \\
$\quad 9(56.25 \%)$ & $13(92.85 \%)$ & 0.039 \\
$\quad$ Yes & $7(43.75 \%)$ & $1(7.14 \%)$ & 0.520 \\
$\quad$ No & $3.78 \pm 2.108$ & $5.23 \pm 2.127$ & 0.520 \\
$\begin{array}{l}\text { Duration of JP drain } \\
\text { placement (days) }\end{array}$ & & & \\
$\begin{array}{l}\text { Duration of IV antibiotics } \\
\quad \text { days) }\end{array}$ & $3.38 \pm 1.310$ & $7.36 \pm 2.706$ & 0.059 \\
$\begin{array}{l}\text { Duration of fasting (days) } \\
\text { Duration of hospitalization } \\
\quad\end{array}$ & $1.90 \pm 1.091$ & $3.5 \pm 3.276$ & 0.000 \\
$\quad$ days) & & & \\
\hline JP $=$ Jackson-Pratt. & & & \\
\hline
\end{tabular}

Table 5. Comparison of postoperative complications between the INT-A and EAR groups

\begin{tabular}{lccc}
\hline & $\begin{array}{c}\text { INT-A group } \\
(\mathrm{n}=16)\end{array}$ & $\begin{array}{c}\text { EAR group } \\
(\mathrm{n}=14)\end{array}$ & $p$ value \\
& $3(18.7 \%)$ & $8(57.1 \%)$ & 0.032 \\
Surgical complication & $1(6.2 \%)$ & $6(42.9 \%)$ & \\
$\quad$ Ileus & & $2(14.3 \%)$ & \\
$\quad$ Wound complications & $1(6.2 \%)$ & & \\
$\quad$ DIC & $1(6.2 \%)$ & & \\
$\quad$ Urinary retention & & $1(7.2 \%)$ & \\
Pneumonia & &
\end{tabular}

DIC $=$ disseminated intravascular coagulation.

compared with $9.21 \pm 3.276$ days in the EAR group $(p=0.520)$.

\section{3) Surgical complications after surgery}

Three out of 16 patients had complications in the INT-A group (18.7\%), significantly lower complication rate than that of EAR group, in which eight of 14 patients (57.1\%) experienced complications $(p=0.032)$.

In the INT-A group, ileus (one case), disseminated intravascular coagulation (one case), and urinary retention (one case) were noted postoperatively, while in the EAR group, six patients $(42.9 \%)$ were diagnosed with postoperative ileus, two patients had wound complications (14.3\%), and one patient developed pneumonia after surgery (Table 5).

\section{4) Analysis of cost-effectiveness}

Cost data were collected during the admission period using the currency of South Korea $(\mathrm{KRW} ; 1,000 \mathrm{KRW}=\$ 0.85$, as of 
June 4,2012$)$. In the INT group, the mean total costs of two hospital admissions were $1,686,000 \pm 940,000 \mathrm{KRW}$. Patients in the EAR group spent $1,506,000 \pm 322,000 \mathrm{KRW}$ during their initial admissions, which was more than the money spent by the INT group ( $p=0.007)$. Increased cost in the INT group was due to interventional surgical drainage procedures which may lead to increase in the total hospital stay in the INT group with $13.33 \pm 5.56$ days, compared with $9.21 \pm 3.378$ days in the EAR group, which was not a significant $(p=0.189)$ (Table 6).

\section{DISCUSSION}

In the previous studies, interval appendectomy did not identify any significant differences in the rate of recurrence, morbidity, or mortality compared with the initial appendectomy (Table 7). However initial nonoperative treatment can be considered as an alternative treatment to initial appendectomy. As shown in Table 7, Yamini et al. first mentioned the advantages of interval laparoscopic appendectomy. In this study, we focused on the postoperative complication, days of hospital stays, cost effectiveness to determine which is more preferable in between initial appendectomy versus interval surgery while developing laparoscopic surgical methods.

Laparoscopic appendectomy in the management of patients with appendiceal masses was first reported by Vargas et al., ${ }^{17}$ who performed laparoscopic interval appendectomy at 6 to 12 weeks after successful conservative treatment in 12 patients. The procedure was conducted successfully and safely in 11 of 12 patients, with a median hospital stay of one day and no perioperative morbidities. The majority of studies of interval appendectomy for the treatment of appendiceal abscess have been retrospective. ${ }^{6-16}$

Nguyen et al. ${ }^{18}$ compared the clinical outcomes of 38 adult patients with appendiceal masses who underwent interval laparoscopic appendectomy with the outcomes of 15 patients who underwent open interval appendectomy. It was found that there was no difference in the operative time between the two groups and that the hospital stay was shorter in the laparoscopic group.

Meshikhes et al. ${ }^{19}$ indicated that the interval appendectomy could be safely omitted after the exclusion of other ileo-cecal pathologies, thus avoiding a second hospital admission and a surgical procedure that is associated with a $10 \sim 20 \%$ complication rate. Emergency laparoscopic appendectomy is emerging as a new safe treatment modality for appendiceal masses, and it may prove to be more cost effective than conservative treatment, even without interval appendectomy, as it is associated with a much shorter hospital stay and obviates the need for long intravenous antibiotic therapy.

In 2012, Zerem et al. ${ }^{20}$ reported that certain appendiceal mass formations could be successfully treated with percutaneous

Table 6. Comparison of the costs of hospital stays between the INT and EAR groups

\begin{tabular}{lccc}
\hline & INT group & EAR group & $p$ value \\
\hline Cost (KRW) & $1,686,000 \pm 940,000^{*}$ & $1,506,000 \pm 322,000$ & 0.007 \\
Duration of hospitalization (days) & $13.33 \pm 5.56$ & $9.21 \pm 3.378$ & 0.189 \\
\hline
\end{tabular}

*Cost of INT group includes initial hospital visit and admission for operation, totally.

Table 7. Results of immediate appendectomy versus interval appendectomy (IA)

\begin{tabular}{|c|c|c|c|c|c|c|c|}
\hline Study & Year & No & $\begin{array}{c}\text { Incidence of } \\
\text { appendiceal mass }\end{array}$ & $\begin{array}{l}\text { Recurrence } \\
\text { rate }\end{array}$ & $\begin{array}{l}\text { Morbidity } \\
\text { of IA }\end{array}$ & Mortality & Comment on IA \\
\hline Bradley [7] & 1978 & 68 & $68 / 2,621(2 \%)$ & $1 / 13(7.6 \%)$ & $19 \%$ & $2 / 68(3 \%)$ & IA suggested \\
\hline Foran [8] & 1978 & 43 & & $4 / 26(15.4 \%)$ & & $1 / 30(3 \%)$ & IA suggested \\
\hline Mosegaard [9] & 1979 & 79 & & $0 / 7$ & $9 / 70(13 \%)$ & & IA not routinely suggested \\
\hline Paull [10] & 1982 & 61 & & $2 / 42(5 \%)$ & $4 / 32(13 \%)$ & $1 / 61(1.6 \%)$ & IA not routinely suggested \\
\hline Skoubo-Kristensen [11] & 1982 & 202 & & $12 / 170(7 \%)$ & $6 / 179(3.4 \%)$ & $1 / 202(0.5 \%)$ & IA suggested \\
\hline Hoffmann [12] & 1984 & 59 & & 9/44 (20.5\%) & & $1.7 \%$ & IA not routinely suggested \\
\hline Bagi [13] & 1987 & 40 & & $2 / 25(8 \%)$ & $4 / 25(16 \%)$ & & IA not routinely suggested \\
\hline Eriksson [14] & 1998 & 38 & & & $5 / 38(13 \%)$ & & IA not routinely suggested \\
\hline Yamini [6] & 1998 & 66 & & $4 \%$ & $10 \%$ & & Laparoscopic IA suggested \\
\hline Willemsen [15] & 2002 & 233 & $233 / 2,325(10 \%)$ & & $36 / 205(17 \%)$ & & IA not routinely suggested \\
\hline Brown [16] & 2003 & 36 & & $2(3 \%)$ & $19(40 \%)$ & & IA suggested \\
\hline
\end{tabular}




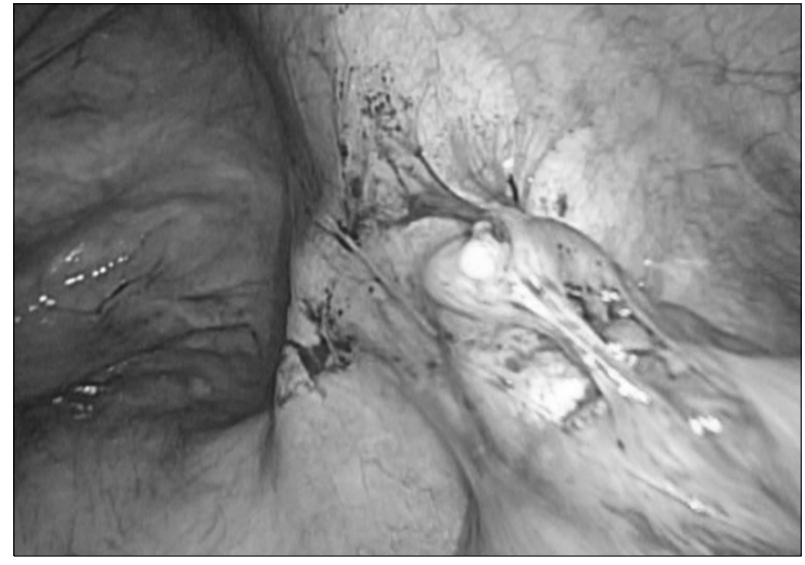

Fig. 2. Laparoscopic view of interval operation for periappendiceal abscess.

catheter drainage, with no need for appendectomy. Since the emergence of laparoscopy, there has been an increase in the percentage of interval appendectomies performed laparoscopically. Laparoscopic appendectomy can be conducted safely and successfully as an early emergency surgery for appendiceal masses, as well as in the interval setting after successful conservative treatment, resulting in short hospital stays and minimal morbidity, analgesia, and scarring. However, information regarding the efficacy and advantages of this treatment modality is limited since most of the studies on this method have a retrospective design.

This was a prospective study of 35 patients who underwent two different treatment methods: an interval laparoscopic approach or initial early appendectomy. In our study, the advantages of interval laparoscopic appendectomy were as follows. First, this technique obviated the need for emergency surgery. In addition, allows surgeons to evaluate the disease more thoroughly, by optimizing the treatment and reducing the complication rates. Second, malignancy (cecal cancer, appendiceal neuroendocrine tumor, or carcinomatosis peritonei) and other diseases (diverticulitis, urachal abscess, tuboovarian abscess, or epithelial hyperplasia) with the similiar clinical features as of appendiceal abscess should be considered in the differential diagnosis of appendiceal masses, as they occur in $10.3 \%$ of cases of appendiceal masses. ${ }^{21}$ Abscesses can mimic the surgeon's primary impression; therefore, interval appendectomy may reduce the incidence of unplanned surgeries. In our study, one carcinomatosis pertionei patient was indentified in INT-A group and from our past experiences elder patients with oncological condition developed periappendiceal abscess. Therefore, particularly in elderly patients, an oncologic evaluation should be considered first, prior to surgery. Third, planned surgery im- proves the possibility that laparoscopic surgery can be utilized, which is a feasible method for treating complicated appendicitis (Fig. 2).

In our study, we attempted interval appendectomy by laparoscopic surgery in all patients except in one patient, who was diagnosed with carcinomatosis peritonei. Laparoscopic interval appendectomy reduces operation time, and attempted to prove that laparoscopic interval appendectomy reduced the rate of conversion compared with initial appendectomy. However, the results showed no conversions in the INT group and one conversion in the EAR group, and the conversion rates were not significantly different. Fourth, there was a shorter fasting period in the INT group. This finding resulted from a low postoperative complication rate and from the minimization of surgery time due to the previous administration of nonoperative treatment. Fifth, the morbidity rate associated with interval laparoscopic appendectomy was less than that associated with early emergency appendectomy (INT group, 18.7\%; EAR group, 57.1\%; $p=0.032$ ).

In contrast, interval laparoscopic appendectomy is higher in the total costs and increase total hospital stays, requiring additional explanations of the advantages and disadvantages of all treatment options offered to patients.

In this study, we undertook only one single-center trial and limited the number of enrolled patients. We calculated the costs of inpatient periods and excluded the costs of outpatient visits, which may have been higher for patients in the INT group because they had more frequent outpatient visits than patients in the EAR group. In this study, there was no recurrence of appendicitis during the follow-up period, even in the 5 patients enrolled in the INT group who did not receive interval appendectomy. Longer follow-up periods are necessary in patients who may develop recurrent appendicitis.

In conclusion, laparoscopic interval appendectomy can result optimal surgical outcomes and may reduce the complication rates as a result of the ability of surgeons to better plan surgeries.

\section{REFERENCES}

1) Townsend Jr. CM, Beauchamp RD, Evers BM, Mattox KL, Sabiston Textbook of Surgery: The Biological Basis of Modern Surgical Practice. 19th ed. Philadelphia: Elsevier Saunders; 2012.

2) Addiss DG, Shaffer N, Fowler BS, Tauxe RV. The epidemiology of appendicitis and appendectomy in the United States. American Journal of Epidemiology 1990;132:910-925. 
3) Flum DR, Koepsell T. The clinical and economic correlates of misdiagnosed appendicitis: nationwide analysis. Archives of Surgery 2002;137:799-804; discussion 804.

4) Flum DR, Morris A, Koepsell T, Dellinger EP. Has misdiagnosis of appendicitis decreased over time? A population-based analysis. JAMA: The Journal of the American Medical Association 2001;286:1748-1753.

5) Semm K. Advances in pelviscopic surgery. Progress in Clinical and Biological Research 1982;112 Pt B:127-149.

6) Yamini D, Vargas H, Bongard F, Klein S, Stamos MJ. Perforated appendicitis: is it truly a surgical urgency? The American Surgeon 1998;64:970-975.

7) Bradley EL, 3rd, Isaacs J. Appendiceal abscess revisited. Archives of Surgery 1978;113:130-132.

8) Foran B, Berne TV, Rosoff L. Management of the appendiceal mass. Archives of Surgery 1978;113:1144-1145.

9) Mosegaard A, Nielsen OS. Interval appendectomy. A retrospective study. Acta Chirurgica Scandinavica 1979;145:109-111.

10) Paull DL, Bloom GP. Appendiceal abscess. Archives of Surgery 1982;117:1017-1019.

11) Skoubo-Kristensen E, Hvid I. The appendiceal mass: results of conservative management. Annals of Surgery 1982;196: 584-587.

12) Hoffmann J, Lindhard A, Jensen HE. Appendix mass: conservative management without interval appendectomy. American Journal of Surgery 1984;148:379-382.

13) Bagi P, Dueholm S, Karstrup S. Percutaneous drainage of appendiceal abscess. An alternative to conventional treatment.
Diseases of the Colon and Rectum 1987;30:532-535.

14) Eriksson S, Styrud J. Interval appendicectomy: a retrospective study. The European journal of surgery = Acta chirurgica 1998;164:771-774; discussion 775.

15) Willemsen PJ, Hoorntje LE, Eddes EH, Ploeg RJ. The need for interval appendectomy after resolution of an appendiceal mass questioned. Digestive Surgery 2002;19:216-220; discussion 221.

16) Brown CV, Abrishami M, Muller M, Velmahos GC. Appendiceal abscess: immediate operation or percutaneous drainage? The American Surgeon 2003;69:829-832.

17) Vargas HI, Averbook A, Stamos MJ. Appendiceal mass: conservative therapy followed by interval laparoscopic appendectomy. The American Surgeon 1994;60:753-758.

18) Nguyen DB, Silen W, Hodin RA. Interval appendectomy in the laparoscopic era. Journal of gastrointestinal surgery: official journal of the Society for Surgery of the Alimentary Tract 1999;3:189-193.

19) Meshikhes AW. Appendiceal mass: is interval appendicectomy "something of the past"? World journal of gastroenterology: WJG 2011;17:2977-2980.

20) Zerem E, Imamovic G, Ljuca F, Alidzanovic J. What is the optimal treatment for appendiceal mass formed after acute perforated appendicitis. World journal of gastroenterology: WJG 2012;18:1849-1850.

21) Lai HW, Loong CC, Chiu JH, Chau GY, Wu CW, Lui WY. Interval appendectomy after conservative treatment of an appendiceal mass. World Journal of Surgery 2006;30:352-357. 